\title{
Thermal transport to droplets on heated superhydrophobic substrates
}

\author{
R. Hays, D. Maynes, J. Crockett* \\ Brigham Young University, Department of Mechanical Engineering, Provo, Utah 84602, \\ USA
}

\begin{abstract}
This paper reports on measurements of thermal transport to solitary sessile water droplets placed on heated superhydrophobic substrates maintained at constant temperature. A single water droplet of nominally $3 \mathrm{~mm}$ diameter is placed on a preheated substrate and allowed to evaporate completely while being imaged from two angles with video cameras and an infrared camera. Substrate temperatures are varied from $60^{\circ} \mathrm{C}$ to $230^{\circ} \mathrm{C}$ and total evaporation time and heat transfer rate are determined. Three different ribpatterned superhydrophobic substrates are investigated of $0.5,0.8$, and 0.95 cavity fraction (relative projected cavity area of substrate to total projected surface area). The rib features range in width from 2 to $30 \mu \mathrm{m}$ and in height from 15 to $20 \mu \mathrm{m}$. Results are also obtained for a smooth hydrophobic substrate for comparison. Droplet evaporation times increase with substrate cavity fraction while overall heat transfer rates decrease. At subcritical substrate temperatures the Nusselt number is larger for lower cavity fraction substrates. For supercritical substrate temperatures, as the cavity fraction increases nucleate boiling is delayed to higher substrate temperatures and the Leidenfrost point occurs at lower temperature. At the highest cavity fraction explored nucleate boiling does not occur at any substrate temperature. At temperatures above the Leidenfrost point the influence of cavity fraction is negligible.
\end{abstract}

Keywords:

superhydrophobic, droplet evaporation, heat transfer, structured substrate

${ }^{*}$ Corresponding author

Email address: crockettj@byu.edu (J. Crockett)

Preprint submitted to International Journal of Heat and Mass Transfer June 5, 2015 


\section{NOMENCLATURE}

$A_{c} \quad$ Projected droplet-substrate contact area

$\overline{A_{p}} \quad$ Average projected area of droplet surface intercepting radiation from substrate

$A_{u} \quad$ Exposed upper-surface area of droplet

$c_{p} \quad$ Specific heat capacity of water

$d_{i} \quad$ Diameter of a single-pixel thick droplet slice from CCD images

$d_{l} \quad$ Width of droplet at droplet-substrate interface longitudinal to ribs

$d_{t} \quad$ Width of droplet at droplet-substrate interface transverse to ribs

$F_{c} \quad$ Substrate cavity fraction

$g \quad$ Acceleration due to gravity

Gr Grashof number

$h_{\text {conv }} \quad$ Convective heat transfer coefficient for external water-air interface

$h_{f g} \quad$ Latent heat of vaporization of water

$h_{p} \quad$ Height of one pixel in CCD images

$h_{s} \quad$ Heat transfer coefficient representing all modes of heat transfer from the substrate to the droplet

$k \quad$ Thermal conductivity of water

$N u \quad$ Nusselt number

$N u_{0} \quad$ Nusselt number for smooth hydrophobic substrate

$N u_{0.5} \quad$ Nusselt number for $F_{c}=0.5$ substrate

$N u_{0.8} \quad$ Nusselt number for $F_{c}=0.8$ substrate

$N u_{0.95} \quad$ Nusselt number for $F_{c}=0.95$ substrate

$\operatorname{Pr} \quad$ Prandtl number

$\dot{q}_{c o n v} \quad$ Convective heat transfer rate at external droplet-air interface

$\dot{q}_{\text {evap }} \quad$ Evaporative heat transfer rate at external droplet-air interface

$\dot{q}_{\text {rad }} \quad$ Radiative heat transfer rate at external droplet-air interface

$\dot{q}_{s} \quad$ Heat transfer rate at droplet-substrate interface

$\dot{q}_{\text {sens }} \quad$ Rate of sensible heat change within droplet

$\overline{\dot{q}}_{s, \text { rad }}$ Average radiative heat transfer rate at droplet-substrate interface

$t \quad$ Time

$t_{e} \quad$ Time at point of critical heat flux

$T_{d} \quad$ Droplet temperature

$T_{s} \quad$ Substrate temperature

$T_{\text {sat }} \quad$ Local saturation temperature of water 


$\begin{array}{ll}T_{\infty} & \text { Ambient air temperature } \\ V & \text { Droplet volume } \\ V_{0} & \text { Volume of spherical droplet with } 3 \mathrm{~mm} \text { diameter } \\ w_{c} & \text { Substrate cavity width } \\ w_{r} & \text { Substrate rib width } \\ \beta & \text { Volumetric thermal expansion coefficient of water } \\ \epsilon & \text { Emissivity of water } \\ \epsilon_{s} & \text { Emissivity of solid substrate } \\ \nu & \text { Kinematic viscosity of water } \\ \theta & \text { Contact angle } \\ \rho & \text { Density of water } \\ \sigma & \text { Stefan-Boltzmann constant }\end{array}$

\section{Introduction}

Evaporation of a water droplet is a common, natural occurrence, yet it is a complex process where both heat and mass transfer occur as the water evaporates into the surrounding unsaturated air. Droplet evaporation from substrates is a regularly studied phenomenon due to its multiple applications including ink-jet printing [5, 34], micro/nano fabrication [6, 50], thin film coatings [23], and spray cooling [20]. Recently, droplet evaporation on superhydrophobic ( $\mathrm{SH}$ ) substrates has captured interest due to the significant alteration of heat transfer dynamics on these substrates caused by very high contact angles and micro/nanoscale surface structuring.

Superhydrophobic (SH) surfaces exhibit internal contact angles with water in excess of $120^{\circ}$ and contact angles up to $177^{\circ}$ have been reported [9]. This non-wetting behavior occurs in nature with such examples as the lotus leaf [2]. Artificial SH surfaces are manufactured by combining micro and/or nanoscale surface roughness with a non-wetting surface coating such as Teflon. Manufacturing methods include, among others, deep reactive ion etching, wax film growth, alumina gel film preparation, and vapor deposition of fluoroalkyl silanes [4]. The left panel of Fig. 1 is an SEM image of a rib/cavity structured surface created using common photolithographic techniques. If the distance between roughness features is minimal, and the Laplace pressure is not exceeded, liquid above the cavities will form menisci between roughness features and rest above the cavities (Cassie-Baxter state $[7]$ ), significantly increasing the contact angle and altering the fluid flow and 

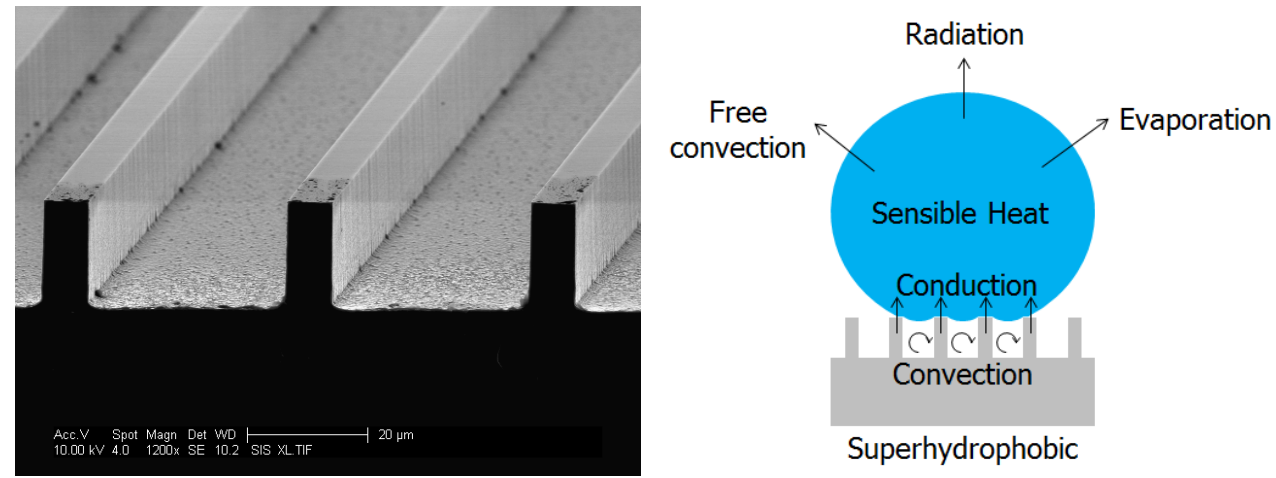

Figure 1: Left panel: SEM image of an $F_{c}=0.8 \mathrm{SH}$ surface. Riight panel: Sessile droplet placed on a heated SH surface (note the ribs are not to scale as the droplet with a $3 \mathrm{~mm}$ diameter would be in contact with hundreds of ribs). Convection, conduction, and radiation (not shown) occur at the base of the droplet, while free convection, radiation, and evaporative cooling occur at the upper surface of the droplet.

heat transfer behavior of liquids on the surface. SH surfaces can be characterized by the cavity fraction, defined as the ratio of the projected area of the cavities to the overall projected area of the surface. Significant research has been focused on the hydrodynamics of fluid interactions with SH surfaces (channel flow [24, 28, 32, 33, 48, 49], jet impingement [21, 29, 36, 37], and droplet motion [38, 40, 45, 47]). More recent research has explored heat transfer dynamics (channel flow [11, 27], condensation [8, 15, 31] and ice formation $[25,46])$. However, much less research has been published regarding the heat transfer behavior with regard to droplet evaporation dynamics on a heated SH substrate [13].

Droplet evaporation rate is a function of the relative humidity of the surrounding air, the surface area in contact with the air (a function of the liquid-solid contact angle), and the substrate temperature and thermal conductivity of the material [14,39]. During evaporation, two distinct modes can occur; constant contact angle and constant contact line, where in the latter the surface area of the droplet in contact with the substrate remains nominally constant $[26,30,35,51]$. During evaporation of droplets from most hydrophilic surfaces the constant contact line mode prevails due to the droplet being pinned to the surface. Pinning may also occur for some hydrophobic and SH surfaces $[10,51]$. However, more commonly hydrophobibc and $\mathrm{SH}$ surfaces exhibit the constant contact angle mode which is characterized by a retreat of the liquid-solid contact line accompanied by the maintenance of 
the liquid-solid interior contact angle.

For a sessile droplet on a smooth hydrophobic surface it has been shown that the evaporation rate is linearly proportional to the radius of the liquidsolid contact area between the droplet and the hydrophobic surface [3, 19]. As hydrophobicity or superhydrophoicity increases, the evaporation rate decreases due to water vapor being trapped near the contact line of the droplet between the sides of the droplet and the substrate [41-44].

When the substrate is heated the evaporation process is enhanced on both hydrophobic and hydrophilic surfaces. Complex circulations due to temperature gradients within the droplet alter the evaporation dynamics and the evaporation rate on hydrophilic surfaces is underestimated when a diffusiononly model is applied $[12,18]$. At surface temperatures greater than the saturation temperature, the dynamics in the liquid change dramatically as nucleate boiling begins to occur. Above the Leidenfrost temperature the vapor layer between the droplet and substrate acts as a barrier to heat transfer resulting in a significant drop in evaporation rates.

For liquid droplets on metal SH surfaces with droplets in the CassieBaxter state there will be regions of high heat transfer (above the microstructure) interspersed with nearly adiabatic regions (above the air cavities). This nonuniform heating due to the droplet sitting atop the microstructured surface significantly decreases the expected droplet temperature and subsequently decreases the evaporation rate [13]. Dash and Garimella created a simple model to estimate the actual interface temperature, which when used with the common vapor-diffusion-only model more accurately estimates the slower evaporation rates observed on $\mathrm{SH}$ surfaces for surface temperatures up to $60^{\circ} \mathrm{C}$ [13]. We expect as the $\mathrm{SH}$ substrate temperature continues to increase the dynamics will be altered even further as the saturation temperature is approached and exceeded. Above the saturation temperature, at supercritical temperatures, heat transfer and droplet boiling dynamics on a SH surface has received only limited prior attention.

In this paper we expand the current understanding of the influence of surface temperature and topography on droplet evaporation and heat transfer rates by characterizing static water droplet evaporation on heated SH surfaces. Experiments are presented for various surface types and over a wide range of surface temperatures. Temporal variation of the droplet geometry and temperature are characterized as a function of the controlling variables. Further, the influence of topography and surface temperature on evaporation rate, interfacial heat flux, droplet boiling behavior, and Leiden- 
frost point temperature are presented.

The remaining sections of the paper are as follows. First the experimental methods used to analyze an evaporating droplet, including hardware used, methods for collection of data, and data analysis are outlined. The next section presents results from the experiments and a quantitative analysis of the heat transfer for various surfaces and temperatures explored. Finally, conclusions are drawn and implications of the work discussed.

\section{Experimental Methodology}

\subsection{Substrates}

A smooth hydrophobic substrate and three different SH substrates manufactured from silicon using photolithographic processes were used. Figure 1 (let panel) shows an SEM image of a rib and cavity microfeatured SH surface created for use in the current research. Rib widths range from 2 to $20 \mu \mathrm{m}$, heights from 15 to $20 \mu \mathrm{m}$, and spacing between the ribs (cavities) ranges from 20 to $38 \mu \mathrm{m}$. All SH surfaces have a thin $(\approx 100 \mathrm{~nm})$ layer of chromium to promote adhesion with Teflon which renders the surfaces superhydrophobic.

A common metric indicating the degree of superhydrophobicity of a given substrate is the cavity fraction, $F_{c}$. It is defined as the relative projected cavity area to total projected area of the surface. For rib and cavity structures this yields

$$
F_{c}=\frac{w_{c}}{w_{c}+w_{r}}
$$

where $w_{c}$ is the width of a cavity and $w_{r}$ is the width of a rib. Cavity fractions of the SH surfaces used here are $F_{c}=0$ (smooth), 0.5, 0.8, and 0.95. For these surfaces, the corresponding initial interior static contact angles with water were approximately $108^{\circ}, 120^{\circ}, 123^{\circ}$, and $140^{\circ}$, respectively.

\subsection{Experimental Setup}

Experiments consist of a sessile distilled water droplet with initial diameter of nominally $3 \mathrm{~mm}$ undergoing evaporation on a heated substrate. Three trials were recorded at each surface temperature ranging from $60^{\circ} \mathrm{C}$ to $230^{\circ} \mathrm{C}$ and for each surface type. A schematic representation of the experimental setup is shown in the left panel of Fig. 2. A patch heater is affixed to the

underside of an aluminum block which is then seated in a block of insulating foam. The heat output of the patch heater is controlled by a voltage transformer. Silicon wafers are affixed to the top of the heated aluminum block 


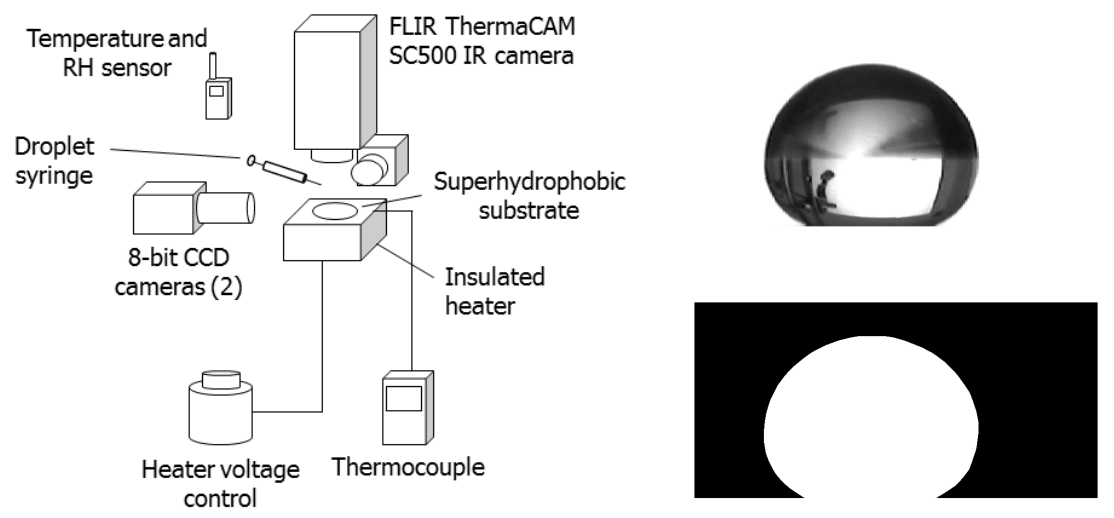

Figure 2: Left panel: Experimental setup including heated substrate apparatus, cameras, and other sensors used for measurement. Right panel: Droplet images directly from the camera (top) and final binary image (bottom).

using thermal paste. A droplet of distilled water is placed on the substrate using a glass syringe with a Teflon needle to minimize particulate contamination from metals which can cause droplets to prematurely pin to the surface during evaporation. Two CCD cameras are used to film the droplet from the sides and a thermal imaging camera captures the temperature of the top of the droplet. In addition, for supercritical substrate temperatures, high speed camera video images of the droplet were captured at 10,000 FPS. High-speed footage was not post-processed to produce quantitative data, but instead was reviewed for qualitative observations of boiling and Leidenfrost behavior.

A K-type thermocouple embedded $3 \mathrm{~mm}$ below the top surface of the aluminum block measured the silicon substrate temperature, $T_{s}$, with an uncertainty of $\pm 0.5^{\circ} \mathrm{C}$. A correlation between the temperature measured by this thermocouple and the true temperature of the upper surface of the substrate was found using a flat-black painted silicon wafer and an infrared camera oriented normal to the surface of the wafer. Using various steady-state temperatures a linear relationship between the thermocouple and infrared camera temperatures was found with a coefficient of determination, $R^{2}=0.9998$.

An infrared camera normal to the substrate surface focused on the top of the evaporating droplet measured the droplet surface temperature, $T_{d}$, through time. This temperature is not to be understood as an average or minimum droplet temperature, but rather a representative droplet temperature. Uncertainty in $T_{d}$ introduced by the infrared camera is nominally 
$\pm 3.2^{\circ} \mathrm{C}$. Additionally the ambient air temperature, $T_{\infty}$, was measured using a thermocouple with an uncertainty of $\pm 0.4^{\circ} \mathrm{C}$.

Two CCD cameras parallel to the substrate surface, one aligned parallel to the microribs on the surface and the other perpendicular, image the geometry of the droplet through time. After filming, a grayscale intensity threshold is determined that distinguishes the droplet from the rest of the image and a binary operation is applied to isolate the droplet as illustrated in the right panel of Fig. 2. Droplet volume was estimated assuming an axisymmetric droplet with a local diameter defined by the length of each horizontal row of pixels within the droplet such that

$$
\Delta V_{i}=\frac{\pi d_{i}^{2} h_{p}}{6}
$$

Here $d_{i}$ is the distance, in physical units, between left and right droplet edges at a particular vertical pixel location, and $h_{p}$ is the height, in physical units, of one pixel. Total droplet volume is then found by summing the individual volumes for each pixel row of the droplet. Any asymmetry in the droplet can be accounted for by averaging together the volumes determined from the two camera images, although the difference in measured volume was always within $\pm 2 \%$.

Projected droplet-substrate contact area can be found by assuming an elliptical droplet footprint on the substrate and using

$$
A_{c}=\frac{\pi d_{l} d_{t}}{4}
$$

where $d_{l}$ and $d_{t}$ are the distance between the edges of the droplet at the droplet-substrate interface for camera views longitudinal and transverse to the microrib structures. An alternative method is to assume the droplet footprint is circular and average the contact areas found using each camera view independently. This results in an order $\pm 0.1 \%$ difference in area. More anisotropic surfaces (higher $F_{c}$ ) yield larger differences in $d_{l}$ and $d_{t}$. For example, the $F_{c}=0.95$ substrate yields a difference between $d_{l}$ and $d_{t}$ of nominally $10 \%$ whereas the smooth hydrophobic substrate yields a difference of nominally $1 \%$.

We note that when placing the droplet on a heated substrate at supercritical temperatures, droplets begin to translate freely, making data collection very difficult. To mitigate this problem, the smooth hydrophobic surface was constructed by forming a slightly concave region in an aluminum plate, 
coated in Teflon, with a large radius of curvature such that changes in contact angle or evaporation dynamics are negligible. Gravity keeps the droplet centered, making data collection feasible. For SH surfaces droplet boiling is much less violent, reducing the need for external stabilization of the droplet. In addition, temporary pinning on ribs stabilizes the droplet, the effects of which are discussed in the Results section.

To estimate the contact angle at the surface an ellipse is fit to the shape of the bottom portion of the droplet in the binary image, below the maximum diameter. At the contact point the slope of the ellipse is calculated, from which the contact angle is computed.

\subsection{Experimental Model}

Heat is exchanged between the substrate and the droplet via conduction through the ribs and convection and radiation through the cavities, as depicted in the right panel of Fig. 1. An overall heat transfer rate from the substrate to the droplet can be estimated by

$$
\dot{q}_{s}=h_{s} A_{c}\left(T_{s}-T_{d}\right)
$$

where $A_{c}$ is the total projected contact area between the droplet and the substrate, $T_{s}$ is the substrate temperature, and $h_{s}$ is an overall convection coefficient representing all modes of heat transfer between the substrate and the droplet through the projected area.

Heat is subsequently transferred from the droplet to the ambient air via convection, radiation, and mass transfer of water vapor. Newton's Law of Cooling can be applied to the free convection between the free surface of the droplet and the surrounding medium to yield

$$
\dot{q}_{\text {conv }}=h_{\text {conv }} A_{u}\left(T_{d}-T_{\infty}\right)
$$

where $\dot{q}_{c o n v}$ is the heat transfer rate due to free convection, $h_{\text {conv }}$ is the free convection coefficient between the droplet free surface and the surrounding medium, $A_{u}$ is the surface area of the droplet, and $T_{\infty}$ is the temperature of the surrounding medium.

Estimating the radiative exchange between the droplet and the surroundings with the Stefan-Boltzmann Law yields

$$
\dot{q}_{\text {rad }}=\epsilon \sigma A_{u}\left(T_{d}^{4}-T_{\infty}^{4}\right)
$$


where $\dot{q}_{\text {rad }}$ is the heat transfer rate due to radiation between the droplet and the surroundings, $\epsilon$ is the emissivity of the droplet free surface, and $\sigma$ is the Stefan-Boltzmann constant.

The heat of evaporation can be modeled by

$$
\dot{q}_{\text {evap }}=h_{f g} \rho \frac{d V}{d t}
$$

where $\dot{q}_{\text {evap }}$ is the heat transfer rate due to evaporation, $h_{f g}$ is the latent heat of vaporization of the droplet liquid, $\rho$ is the density of the droplet liquid, and $d V / d t$ is the time rate of change of droplet volume.

Sensible heat change within the droplet can be modeled by

$$
\dot{q}_{\text {sens }}=c_{p} \rho V \frac{d T_{d}}{d t}
$$

where $\dot{q}_{\text {sens }}$ is the energy storage rate due to sensible heat, $c_{p}$ is the specific heat capacity of the droplet liquid, $V$ is the droplet volume, and $d T_{d} / d t$ is the time rate of change of droplet temperature. An energy balance on the drop requires that $\dot{q}_{s}=\dot{q}_{\text {conv }}+\dot{q}_{\text {rad }}+\dot{q}_{\text {evap }}+\dot{q}_{\text {sens }}=\dot{q}_{\text {tot }}$.

A representative analysis of the relative importance of the four modes of heat transfer outlined in (5) to (8) is shown in Fig. 3. These are results of a single experiment but are representative of all experiments conducted. Heat transfer rates are normalized by the overall heat transfer rate and shown as a function of time normalized by total evaporation time. An initial heat-up period exists where heat transfer due to sensible heat is significant. However a quasi-steady state is achieved rapidly and the heat transfer due to mass transfer of water vapor dominates all other terms, representing greater than $95 \%$ of the total heat loss. Consequently, the heat transfer due to convection and radiation from the upper surface of the droplet along with the sensible heat change within the droplet are neglected in the subsequent results and analysis.

Conservation of energy for the evaporating droplet in the quasi-steady state regime can now be approximated with a balance of heat transfer from the surface to the droplet (4) and droplet evaporation (7)

$$
h_{s} A_{c}\left(T_{s}-T_{d}\right)=h_{f g} \rho \frac{d V}{d t}
$$

Thus, the total heat transfer to the droplet can be estimated by measuring the heat lost to evaporation and $h_{s}$ can be determined as,

$$
h_{s}=\frac{h_{f g} \rho d V / d t}{A_{c}\left(T_{s}-T_{d}\right)}
$$




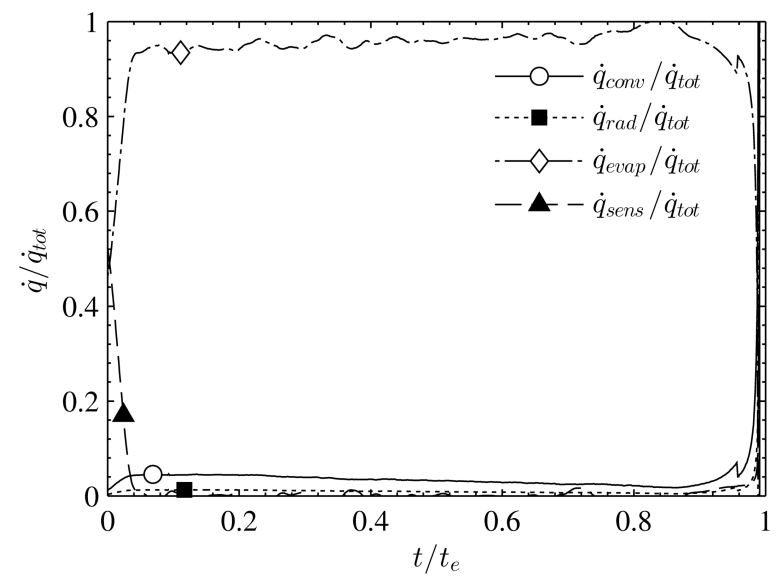

Figure 3: Ratio of each mode of heat transfer to the total heat loss as a function of time normalized by the total evaporation time for a representative scenario.

\section{Results}

First we present qualitative results which capture the nature of droplet boiling and vaporization at temperatures greater than the saturation temperature. Then we quantify the droplet volume, contact angle and area, and total phase change time. Next, heat transfer rates are presented. Finally, Nusselt number relations are presented for the scenarios with subcritical substrate temperatures $(N u$ cannot be quantified at supercritical temperatures due to the boiling and bouncing of the droplets rendering contact area calculations impossible).

\subsection{Qualitative Observations at Supercritical Substrate Temperatures}

High-speed images of droplets resting on the smooth hydrophobic surface for a range of substrate temperatures are shown in Fig. 4. Frames were sampled at $0.0005 \mathrm{~s}$ intervals. In the top row, the surface temperature is approximately $85^{\circ} \mathrm{C}$, which is $10^{\circ} \mathrm{C}$ below the local saturation point of water, $T_{\text {sat }}=95.0^{\circ}$. Boiling is notably absent, as expected. The second row of the figure shows the surface at approximately $105^{\circ} \mathrm{C}$. This is near the onset of nucleate boiling, and the droplet shows signs of boiling with observable surface agitation. In the third row of images, the surface is at approximately $125^{\circ} \mathrm{C}$, near the critical heat flux temperature. Surface agitation is most pronounced in this set of images, thus it is expected the maximum heat transfer rate is near this substrate temperature. Droplet bouncing also occurs at this 


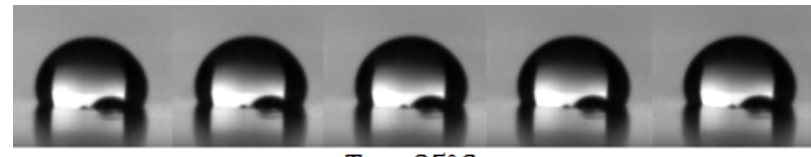

$T_{s} \approx 85^{\circ} \mathrm{C}$
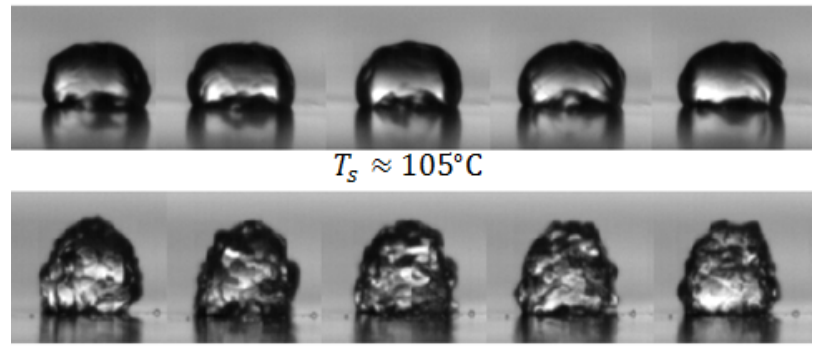

$T_{s} \approx 125^{\circ} \mathrm{C}$

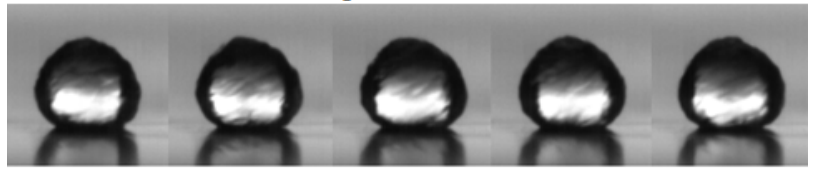

$T_{s} \approx 180^{\circ} \mathrm{C}$

Figure 4: High speed images of droplet evaporation on the smooth hydrophobic surface $\left(F_{c}=0\right)$ at various supercritical substrate temperatures. Images were taken at $0.0005 \mathrm{~s}$ intervals. Each row is from a different substrate temperature, as noted.

temperature. The last row shows the surface at a much higher substrate temperature, $180^{\circ} \mathrm{C}$. Notably, the surface agitation is lessened as the droplet nears the Leidenfrost point at nominally $200^{\circ} \mathrm{C}$, where these surface agitations are expected to die out completely as a stable vapor layer forms between the droplet and the substrate.

High-speed imaging also provides visual evidence that heat transfer to the droplet decreases with increasing cavity fraction. Figure 5 shows a series of frames at $0.0005 \mathrm{~s}$ intervals from each substrate at $125^{\circ} \mathrm{C}$. The top row is recreated from the third row of Fig. 4. Again note the presence of rapid droplet surface agitation, indicative of nucleate boiling. Surface agitation markedly decreases in the second row where $F_{c}=0.5$. In the third row, $F_{c}=0.8$, only a small degree of surface agitation is still present and nucleate boiling is notably absent. Finally, in the bottom row of images which show a droplet evaporating on the $F_{c}=0.95$ surface, no surface agitation is present, indicating a tremendous reduction in heat transfer relative to the smooth and lower cavity fraction cases. 


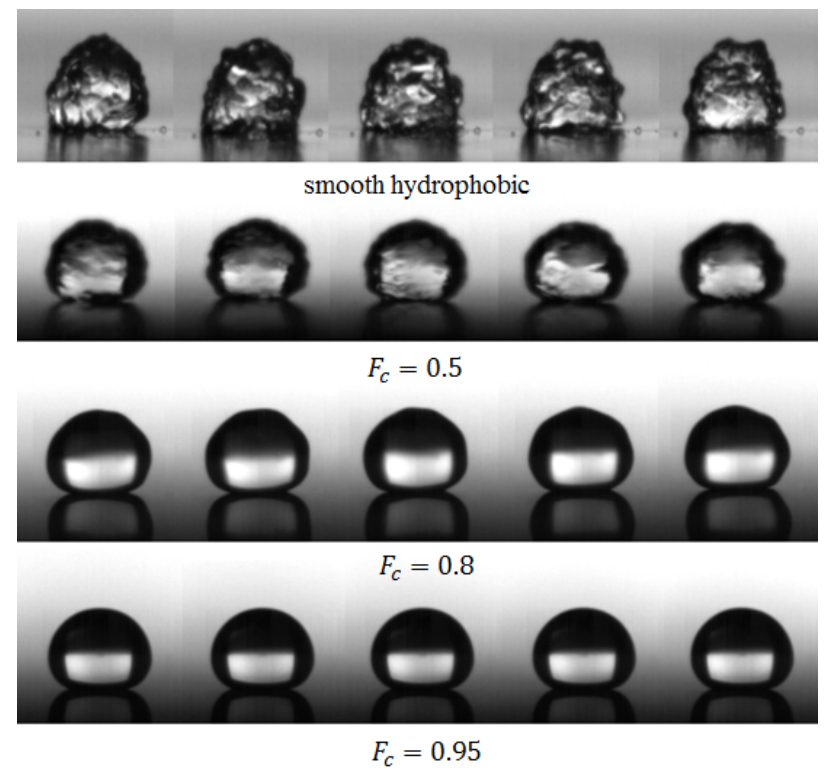

Figure 5: High speed images of droplet evaporation at $T_{s} \approx 125^{\circ} \mathrm{C}$. Images were taken at $0.0005 \mathrm{~s}$ intervals. Each row is from a different substrate type: smooth hydrophobic (top), $F_{c}=0.5$ (second), $F_{c}=0.8$ (third), and $F_{c}=0.95$ (bottom).

\subsection{Droplet Evaporation Dynamics}

Droplet temperature as a function of time for a single test case of each substrate is shown in Fig. 6. These results are for $T_{s}=80^{\circ} \mathrm{C}$ but are representative of all cases where the substrate temperature is less than the water saturation temperature. There is an initial period of nominally $10 \mathrm{~s}$ during which the temperature quickly rises followed by a relatively long period of modestly declining droplet temperature. This period is the primary focus of the current research. In general, $T_{d}$ decreases with increasing $F_{c}$, which is the same trend observed by Dash and Garimella at lower surface temperatures [13]. Near the end of the droplet evaporation process, $T_{d}$ experiences drastic changes caused by rapid vaporization as the droplet vanishes.

Projected droplet-substrate contact areas as a function of time, for the same representative case of Fig. 5, are shown in the left panel of Fig. 7. Note the contact area decreases nearly linearly with time which is analogous to the $D^{2}$ law of suspended evaporating droplets [17]. If the substrate is unheated, the driving force for evaporation is the difference in water concentration between the droplet surface and the ambient air [16]. As the substrate is heated, however, the substrate temperature itself becomes the important 


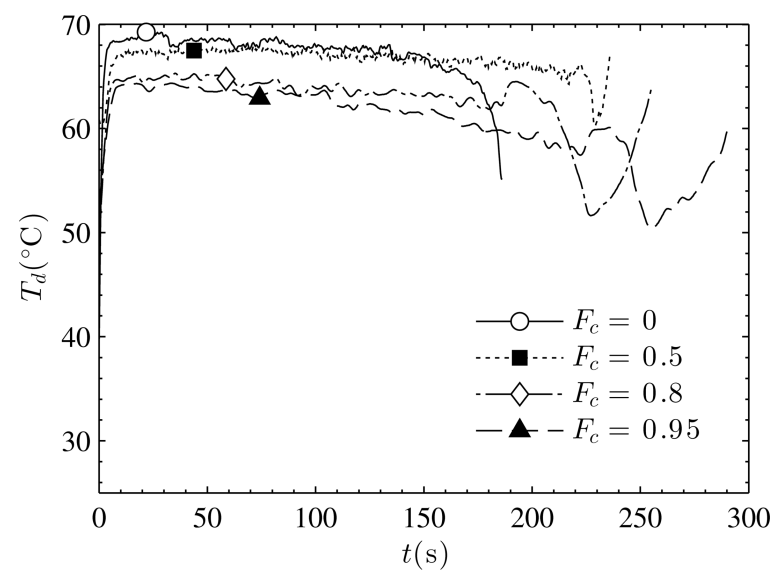

Figure 6: Transient droplet temperature as a function of time for substrate temperature of nominally $80{ }^{\circ} \mathrm{C}$ and for all substrates considered.

variable in droplet evaporation. Adding heat to the evaporating droplet serves to accelerate the process, but the effects of evaporative cooling keep the droplet temperature well below that of the substrate.

Inspection of the lines in Fig. 7 show a step-wise fashion of contact area decrease in some regions which is due to the pinning phenomenon described previously. For SH surfaces, this is explained physically by temporary pinning at a microrib until surface tension overcomes the pinning forces and the droplet then retracts and pins at a different rib. For the smooth hydrophobic substrate pinning likely occurs at imperfections on the surface resulting in unpredictable pinning behavior.

Droplet pinning and unpinning can also be observed in the contact angle, $\theta$, dynamics shown in the right panel of Fig. 7. This is most prominent in the smooth hydrophobic case. For all surfaces the contact angle remains relatively constant over most of the evaporation process until the end of the droplet's life, when the droplet becomes pinned to the surface and contact angle decreases rapidly. This behavior is typical across all substrate types and temperatures, when boiling is not present, and is not dependent on substrate temperature. Studies of droplet evaporation have shown similar results of pinning and unpinning dependent on droplet size and surface structure $[1$, 10, 22, 30, 42, 51]. Specifically, Jung and Bhushan found variations in contact angle for microscale droplet evaporation due to changes in the state of the droplet from Cassie-Baxter to Wenzel [22]. However, in this paper we are focused on large droplets in the regime of nearly constant $\theta$ and $T_{d}$, and thus 

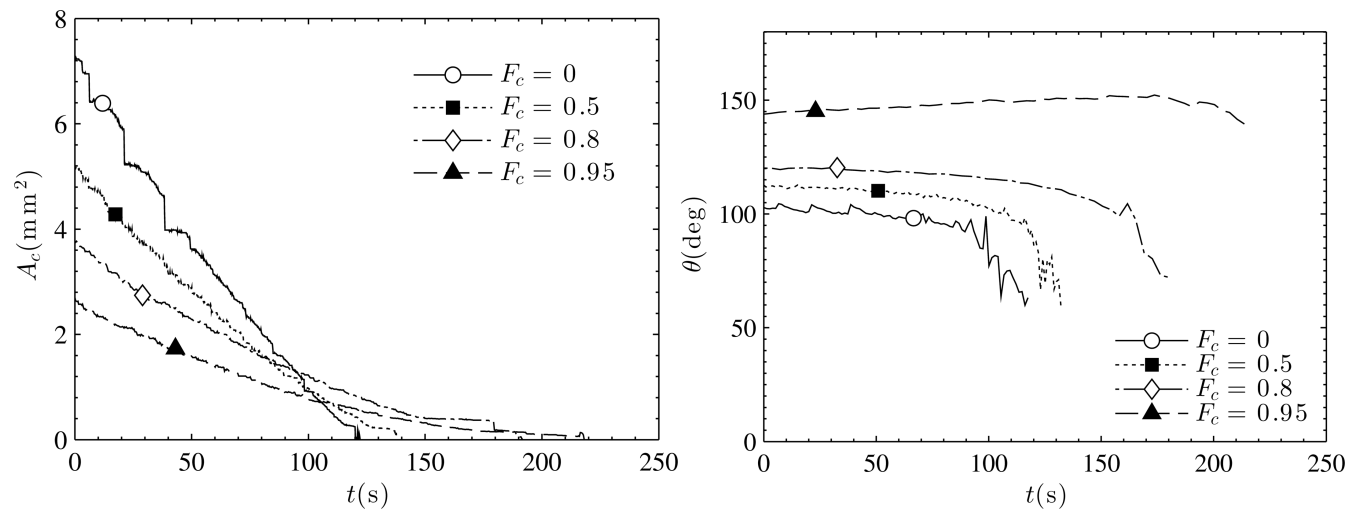

Figure 7: Left panel: Projected droplet-substrate contact area as a function of time for substrate temperature of nominally $80{ }^{\circ} \mathrm{C}$ and for all substrates considered. Right panel: Internal droplet-substrate contact angle as a function of time for substrate temperature of nominally $80^{\circ} \mathrm{C}$ and for all substrates considered.

the varying $\theta$ region at the end of the droplet life is not considered further.

Of particular note in the left panel of Fig. 7 is the difference in initial contact area between the various substrates. As $F_{c}$ increases, $\theta$ increases (right panel), and the initial $A_{c}$ decreases. This in part explains the increased droplet lifetimes with increasing $F_{c}$, since less heat is transferred from the substrate to the droplet as $A_{c}$ becomes smaller.

Temporal droplet volume $(V)$ data (smoothed) for the same representative cases depicted in Fig. 7, are shown in the left panel of Fig. 8. Results at a higher substrate temperature of $T_{s}=120^{\circ} \mathrm{C}$ are shown in the right panel. Volume data obtained for droplets in the nucleate and transitional boiling regimes tends to be very noisy due to rapid droplet oscillations. Thus all volume data were smoothed with a weighted, third degree polynomial, linear least squares regression, localized over no less than $1 / 4$ of the span of the $V$ data, and with $R^{2}=0.9999$. All data here and in subsequent figures was truncated by defining $t=0$ as the condition when the droplet volume, $V_{0}$ is equivalent to that of a sphere with a $3 \mathrm{~mm}$ diameter.

As expected, the evaporating droplet volume decreases continuously with time. Additionally, the rate of volume decrease, $d V / d t$, also decreases as evaporation progresses. This general behavior was typical for all substrate types and temperatures. However, of note is the decrease in initial $d V / d t$ as $F_{c}$ increases, concomitant with a decrease in direct liquid-solid contact and leading to increased droplet lifetimes. 

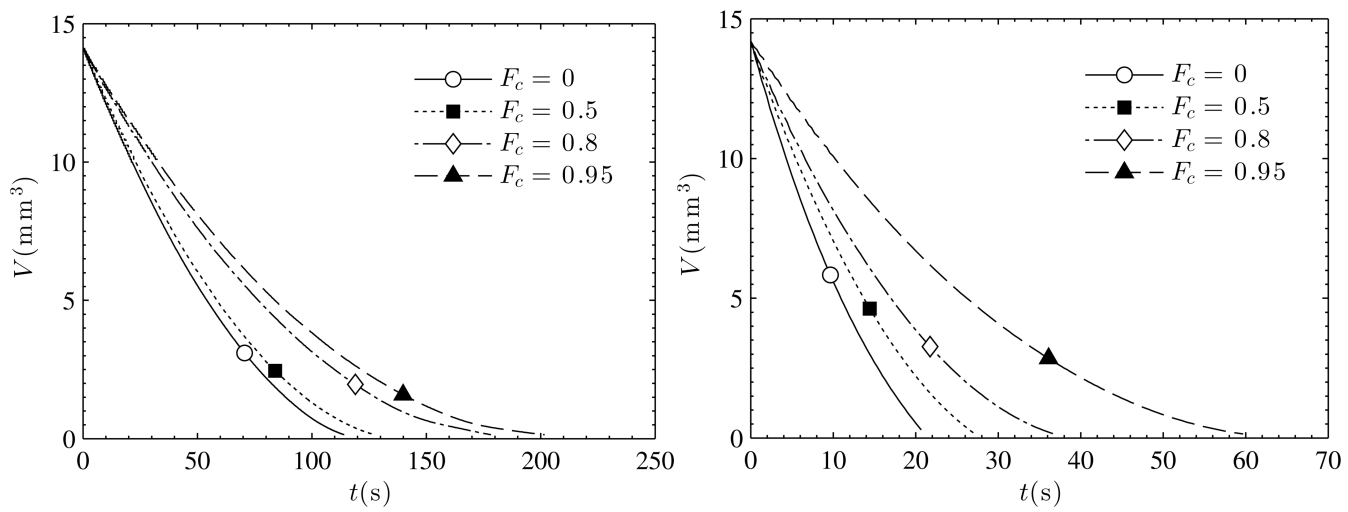

Figure 8: Droplet volume as a function of time for all substrates considered for $T_{s}=80^{\circ} \mathrm{C}$ (left panel) and $T_{s}=120^{\circ} \mathrm{C}$ (right panel).

Total evaporation times for droplets on each substrate as a function of substrate temperature are shown in Fig. 9, where evaporation time is defined as the time for a droplet to reach $10 \%$ of the initial volume. Three tests at each substrate temperature were averaged for the results shown. In the subcritical regime (left panel), as the substrate temperature increases, the evaporation time decreases due to increased heat transfer. The evaporation time also increases as $F_{c}$ increases due to the decrease in both projected droplet-substrate contact area and physical droplet-substrate contact. The average decrease in evaporation times for the droplets on the $\mathrm{SH}$ surfaces compared to the smooth surface is $13 \%, 28 \%$, and $40 \%$ for the $F_{c}=0.5,0.8$, and 0.95 surfaces, respectively. Power-law curve-fits for each substrate were determined and are shown in the left panel of Fig. 9. Of note is the observation that power-law curve fits intersect at about $25^{\circ} \mathrm{C}$ (ambient temperature) when extrapolated to the left of the figure, as expected.

At supercritical substrate temperatures (right panel of Fig. 9) the trend of decreasing vaporization time with increasing substrate temperature continues until it reaches a minimum at around $T_{s}=120^{\circ} \mathrm{C}$. This minimum in $t_{e}$ corresponds to the critical heat flux point where heat input reaches a maximum. It is easily observed in the smooth substrate data whereas the $F_{c}=0.95$ substrate data shows much less variation. Beyond the critical heat flux point evaporation time increases slightly corresponding to the transition from nucleate to film boiling (which is notably absent in the $F_{c}=0.95$ scenario). Above $T_{s} \approx 160^{\circ} \mathrm{C}$ there is no clear distinction in $t_{e}$ between the hydrophobic and $\mathrm{SH}$ substrates. 

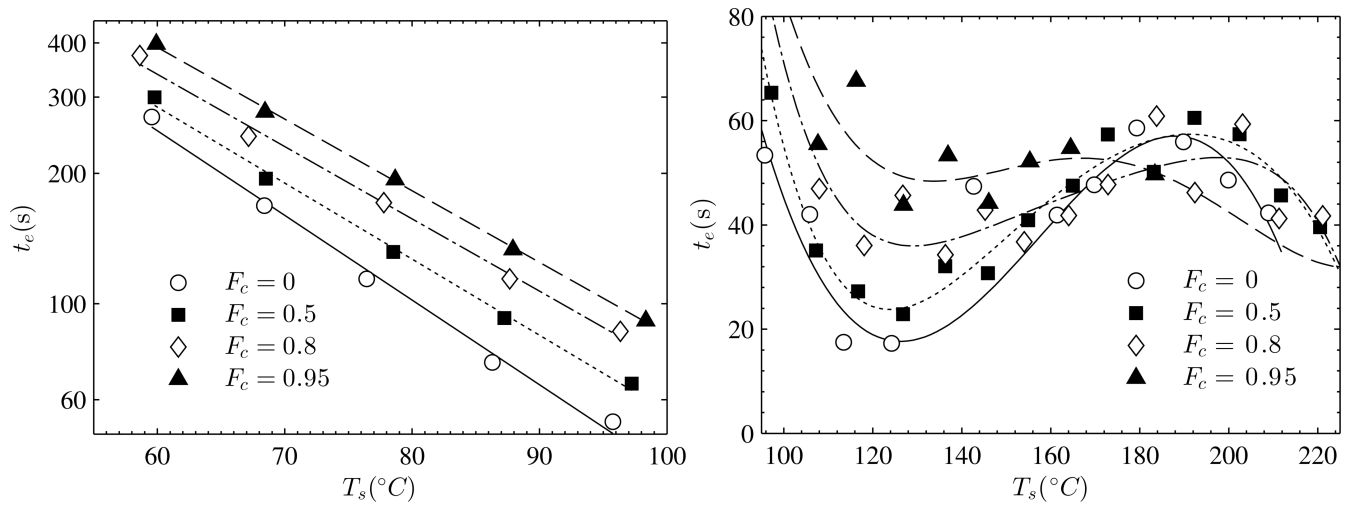

Figure 9: Droplet evaporation time as a function of substrate temperature for all substrates considered and for all experimental runs. The left panel shows the evaporation time (log scale) for subcritical substrate temperatures as well as power-law curve fits. The right panel shows the evaporation time for supercritical substrate temperatures with polynomial fits to the experimental data.

\subsection{Heat Transfer Rates}

Total heat input is determined from (7) and averaging over the time domain corresponding to $V_{0} \geq V \geq 0.1 V_{0}$. This resultant average heat input, $\overline{\dot{q}_{s}}$, is shown for subcritical substrate temperatures in Fig. 10 and supercritical temperatures in the left panel of Fig. 11 as a function of the surface temperature. Faired lines are also shown to view the data for each $F_{c}$ more clearly. Additionally the right panel of Fig. 11 includes droplet images corresponding to each substrate for various temperatures defined by the ranges shown.

In the subcritical regime shown in Fig. 10 for all substrates $\bar{q}_{s}$ increases with increasing $T_{s}$. As $F_{c}$ increases $\overline{\dot{q}_{s}}$ decreases. The average decrease in the total heat transfer rates to the droplets on the SH surfaces (compared to the smooth surface) is $20 \%, 35 \%$, and $45 \%$ for the $F_{c}=0.5,0.8$, and 0.95 surfaces, respectively. In the supercritical regime(left panel of Fig. 11) $\overline{\dot{q}_{s}}$ continues to increase with $T_{s}$ until it reaches a maximum, near $T_{s}-T_{s a t} \approx 20^{\circ} \mathrm{C}$ for the smooth substrate. This point corresponds well to the critical heat flux point in the classical boiling curve. Note the corresponding droplet image for $F_{c}=0$ in region $\mathrm{B}$ in the right panel of Fig. 11 where rapid boiling is evident. Further increasing $T_{s}$ on the smooth surface decreases the average heat input steadily until reaching a minimum near $T_{s}-T_{s a t} \approx 90-100^{\circ} \mathrm{C}$, corresponding to the Leidenfrost point. 


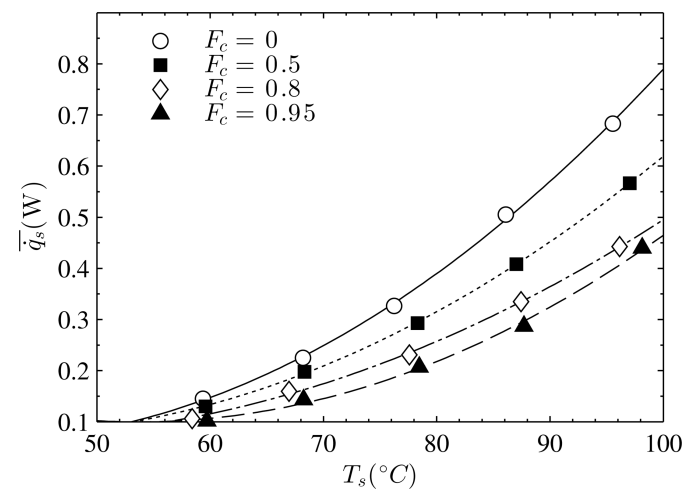

Figure 10: Time-averaged droplet-substrate heat transfer rate as a function of substrate temperature, in the subcritical regime, for all substrates studied.
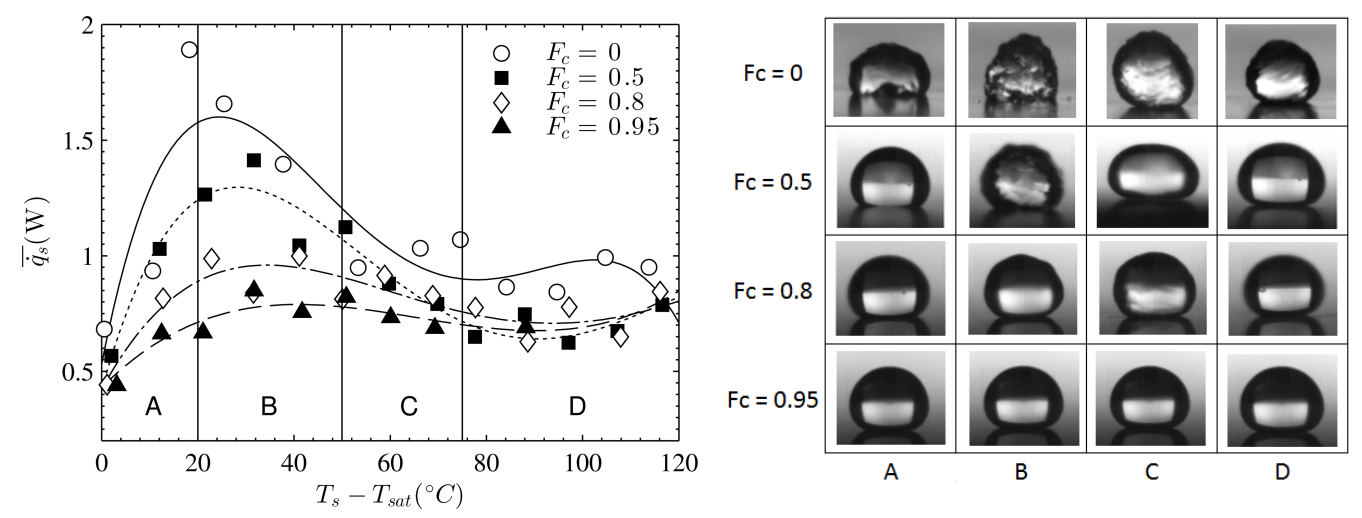

Figure 11: Time-averaged droplet-substrate heat transfer rate as a function of substrate temperature, in the supercritical regime, for all substrates studied. Note in the left panel the average heat transfer rate is plotted against $T_{s}-T_{\text {sat }}$. Regions $\mathrm{A}, \mathrm{B}, \mathrm{C}$, and $\mathrm{D}$ correspond to temperature ranges for which images of the heated droplets are shown in the right panel for each substrate. 
SH substrates exhibit some important differences from the smooth substrate. First, with increasing $F_{c}$ the average heat input decreases for all temperatures below the Leidenfrost point temperature. This is most evident in Fig. 11 near $T_{s}-T_{\text {sat }} \approx 20-30^{\circ} \mathrm{C}$ where nucleate boiling is occurring on the smooth and lower $F_{c}$ substrates (see the corresponding snapshot of droplet in right panel) but not on the $F_{c}=0.95$ substrate. Here the maximum heat transfer rate decreases from $1.9 \mathrm{~W}$ on the smooth surface to $1.4,0.95$, and 0.8 $\mathrm{W}$ on the $F_{c}=0.5,0.8$, and 0.95 surfaces respectively. Thus the air cavities of the SH surface not only hinder heat transfer at all substrate temperatures, but also dramatically alter the dynamics of nucleate boiling. Second, the images shown in the right panel of Fig. 11, show much less convection for the high $F_{c}$ substrates at $T_{s}-T_{s a t} \approx 30^{\circ} \mathrm{C}$ or above and it seems nucleate boiling can be greatly minimized or eliminated when a SH substrate is employed.

We note here that the average rate of heat transfer to the droplet, $\overline{\dot{q}}_{s}$, is comprised of contributions from convection between the solid substrate and the liquid droplet and conduction and radiation through the air in the cavity regions. It can be estimated that radiation contributes

$$
\overline{\dot{q}_{s, r a d}}=\epsilon_{s} \sigma \overline{A_{p}}\left(T_{s}^{4}-T_{d}^{4}\right)
$$

where $\epsilon_{s}$ is the emissivity of the solid surface and $\overline{A_{p}}$ is the average projected area of the droplet surface that intercepts radiation from the solid surface below. If we assume the solid surface acts as a blackbody, $\epsilon_{s}=1 . \overline{A_{p}}$ can be approximated as $\pi{\overline{D_{d}}}^{2} / 4$, where $\overline{D_{d}}$ is the average horizontal diameter of the sessile droplet. In this case, $\overline{D_{d}} \approx 1.5 \mathrm{~mm}$. Using the extreme case of $T_{s}=100^{\circ} \mathrm{C}$ and $T_{d}=70^{\circ} \mathrm{C}, \overline{\dot{q}_{s, r a d}}$ computes to $0.5 \mathrm{~mW}$, which is three orders of magnitude smaller than the contributions of conduction and convection. Further, the conduction through the air layer can be estimated and is nominally $10-20 \mathrm{~mW}$, also much smaller than the measured values. Thus the transport is dominated by convection from the solid ribs into the liquid.

\subsubsection{Nusselt Number Analysis for Subcritical Substrate Temperatures}

Grashof number, $G r$, Nusselt number, $N u$, and Prandtl number, $P r$ were calculated for all data sets at subcritical substrate temperatures. $G r$ is defined here as

$$
G r=\frac{g \beta\left(T_{s}-T_{d}\right) V}{\nu^{2}}
$$

where $g$ is the acceleration due to gravity, and $\beta$ and $\nu$ are the volumetric thermal expansion coefficient and kinematic viscosity of the liquid, respec- 
tively. Here the cube root of droplet volume was chosen as the characteristic length scale. $\mathrm{Nu}$ is defined here as

$$
N u=\frac{h_{s} A_{c}^{1 / 2}}{k}
$$

where $k$ is the thermal conductivity of the liquid and the square root of the projected droplet-substrate contact area was used as the characteristic length scale for $N u$, which represents the effective area through which convection occurs. $\operatorname{Pr}$ is defined as

$$
\operatorname{Pr}=\frac{\rho c_{p} \nu}{k}
$$

We note that all fluid properties in (12) to (14) were determined at the droplet temperature.

For laminar free convection on a (vertical flat plate the Nusselt number is a function of $G r^{1 / 4}$ and $\operatorname{Pr}^{1 / 3}$. Thus, $N u$ as a function of $G r^{1 / 4} \operatorname{Pr}^{1 / 3}$ for the $F_{c}=0.95$ substrate is shown in the left panel of Fig. 12. A least-squares power-law curve fit of the data is superimposed. Although the spread is as great as $30 \%$ above and $20 \%$ below the curve, this fit allows comparison among the substrates, which are shown in the right panel of Fig. 12. Equations defining each of these lines are

$$
\begin{gathered}
N u_{0}=2.53 G r^{1 / 4} \operatorname{Pr}^{1 / 3} \\
N u_{0.5}=2.40 G r^{1 / 4} \operatorname{Pr}^{1 / 3} \\
N u_{0.8}=2.14 G r^{1 / 4} \operatorname{Pr}^{1 / 3} \\
N u_{0.95}=1.65 G r^{1 / 4} \operatorname{Pr}^{1 / 3}
\end{gathered}
$$

where the subscripts represent the $F_{c}=0,0.5,0.8$, and 0.95 substrates.

In general, $N u$ increases with $G r^{1 / 4} \mathrm{Pr}^{1 / 3}$ as expected. Further, for a given $G r, N u$ is larger for smaller $F_{c}$. Nu decreases modestly (nominally $5 \%$ ) for cavity fractions up to 0.5 but drops off dramatically as cavity fraction is further increased. For a cavity fraction of $0.8, N u$ is decreased by nominally $15 \%$ from the smoooth hydrophobic case, and for a cavity fraction of $0.95, N u$ is decreased by nominally $35 \%$. It should be noted that the definition of $N u$ employed here accounts for the decrease in droplet contact area that prevails for the different substrates through the use of $A_{c}^{1 / 2}$ as the characteristic length scale. Thus, comparison of $N u$ between the different surface types yields the amount of deviation in the convection coefficient that prevails due only to 

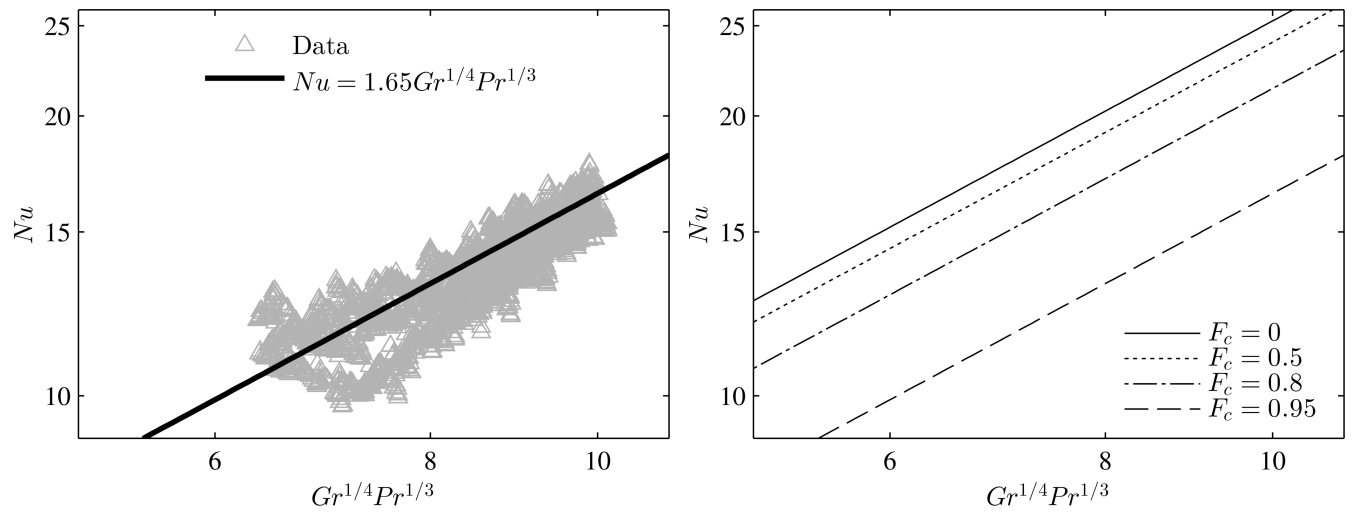

Figure 12: $N u$ as a function of $G r^{1 / 4} \mathrm{Pr}^{1 / 3}$. In the left panel experimental data and a power-law curve fit for the $F_{c}=0.95$ substrate are shown. In the right panel are power-law curve-fits for all substrates studied. Note the log-scale of the axes.

the trapped air in the cavity regions. Of course, the combined effect of more trapped air and smaller $A_{c}$ as $F_{c}$ increases combine to yield overall heat transfer rates that show greater deviation between surfaces, as illustrated in Fig. 10.

\section{Conclusion}

This paper has reported on experiments characterizing droplet-substrate convective heat transfer for sessile water droplets resting on rib-patterned superhydrophobic substrates. At a given substrate temperature, droplet temperatures tend to be lower for substrates with higher cavity fraction. This corresponds to decreased evaporation rates for subcritical substrate temperatures. These trends are explained by both the increase in contact angle and decreased physical contact between the droplet and the substrate due to the air cavities leading to lower projected total droplet-substrate contact areas.

At surface temperatures higher than the saturation temperature, increasing the cavity fraction decreases the heat transfer rate, delays the onset of nucleate boiling, and decreases the temperature where Leidenfrost behavior occurs. If the cavity fraction is increased enough $\left(F_{c}=0.95\right.$ for this paper), nucleate boiling is suppressed at all surface temperatures and the droplet exhibits Leidenfrost-like behavior near the classical critical heat flux point. This change may be explained by the presence of persistent vapor cavities on the SH surfaces. These cavities act to stabilize the vapor film beneath the 
boiling droplet at lower supercritical temperatures.

Nusselt number data confirm that decreased heat flux exists for droplets on substrates of greater cavity fraction, with the effect increasing with increasing $F_{c}$. Near $F_{c}=0.95$, even small changes in cavity fraction exhibit appreciable changes in $N u$. Data presented here allow for the prediction of effective droplet-substrate heat transfer coefficients over a wide range of cavity fractions.

\section{Acknowledgement}

This material is based upon work supported by the National Science Foundation under Grant No. (CBET-1235881).

\section{References}

[1] Anantharaju, N., Panchagnula, M., Neti, S., 2009. Evaporating drops on patterned surfaces: Transition from pinned to moving triple line. Journal of Colloid and Interface Science 337 (1), 176-182.

[2] Barthlott, W., Neinhuis, C., April 1997. Purity of the sacred lotus, or escape from contamination in biological surfaces. Planta 202 (1), 1-8.

[3] Birdi, K., Vu, D., Winter, A., May 1989. A study of the evaporation rates of small water drops placed on a solid surface. The Journal of Physical Chemistry 93 (9), 3702-3703.

[4] Blossey, R., May 2003. Self-cleaning surfaces-virtual realities. Nature Materials 2 (5), 301-306.

[5] Calvert, P., 2001. Inkjet printing for materials and devices. Chem. Mater. 13 (10), 3299-3305.

[6] Carroll, G. T., Wang, D., Turro, N. J., Koverstein, J. T., 2006. Photochemical micropatterning of carbohydrates on a surface. Langmuir 22 (6), 2899-2905.

[7] Cassie, A., Baxter, S., June 1944. Wettability of porous surfaces. Transactions of the Faraday Society 40, 546-551. 
[8] Chen, C. H., Cia, Q., Tsai, C., Chen, C. L., Xiong, G., Yu, Y., Ren, Z., 2007. Dropwise condensation on superhydrophobic surfaces with twoteir roughness. Applied Physics Letters 90, 173108.

[9] Chen, W., Fadeev, A., Hsieh, M., Oner, D., Youngblood, J., McCarthy, T., April 1999. Ultrahydrophobic and Ultralyophobic Surfaces: Some Comments and Examples. Langmuir 15 (10), 3395-3399.

[10] Choi, C. H., Kim, C. J., 2009. Droplet evaporation of pure water and protein solution on nanostructured superhydrophobic surfaces of varying heights. Langmuir 25 (13), 7561-7567.

[11] Cowley, A., Maynes, D., Crockett, J., 2014. Effective temperature jump length and influence of axial conduction for thermal transport through channels with superhydrophobic walls. International Journal of Heat and Mass Transfer 79, 573-583.

[12] Crafton, E. F., Black, W. Z., 2004. Heat transfer and evaporation rates of small liquid droplets on heated horizontal surfaces. International Journal of Heat and Mass Transfer 47, 1187-1200.

[13] Dash, S., Garimella, S. V., 2014. Droplet evaporation on heated hydrophobic and superhydrophobic surfaces. Physical Review E 89, 042402 .

[14] David, S., Sefiane, K., Tadrist, L., 2007. Experimental investigation of the effect of thermal properties of the substrate in the wetting and evaporation of sessile drops. Colloids Surf. A 298, 108-114.

[15] Dietz, C., Rykaczewski, K., Fedorov, A. G., Joshi, Y., 2010. Visualization of droplet departure on a superhydrophobic surface and implications to heat transfer enhancement during dropwise condensation. Applied Physics Letters 97, 033104.

[16] Fick, A., 1855. Ueber diffusion. Annalen der Physik 170 (1), 59-86.

[17] Godsave, G., 1953. Studies of the combustion of drops in a fuel spray-the burning of single drops of fuel. Symposium (International) on Combustion 4 (1), 818-830. 
[18] Granadas, L., Reynard, C., Santini, R., Tadrist, L., 2005. Experimental study of the evaporation of a sessile drop on a heat wall. Int. J. Therm. Sci. 44 (2), 137-146.

[19] Grandas, L., Santini, R., Tadrist, L., February 2004. Heat Transfer Induced by Evaporation of a Sessile Drop: Influence of Wetting Surface. AIP Conference Proceedings 699, 156-163.

[20] Jia, W., Qiu, H. H., 2003. Experimental investigation of droplet dynamics and heat transfer in spray cooling. Experimental Thermal and Fluid Science 27, 829-838.

[21] Johnson, M., Maynes, D., Crockett, J., 2014. Experimental characterization of hydraulic jump caused by jet impingement on micro-patterned surfaces exhibiting ribs and cavities. Experimental Thermal and Fluid Science 58, 216-233.

[22] Jung, Y. C., Bhushan, B., 2007. Wetting behaviour during evaporation and condensation of water microdroplets on superhydrophobic patterned surfaces. Journal of Microscopy 229, 127-140.

[23] Kimura, M., Misner, M. J., Xu, T., Kim, S. H., Russell, T. P., 2003. Long-range ordering of diblock copolymers induced by droplet pinning. Langmuir 19, 9910.

[24] Lee, C., Choi, C. H., Kim, C. J., 2008. Structured surfaces for giant liquid slip. Physical Review Letters 101, 064501.

[25] Liu, Z., Gou, Y., Wang, J., Cheng, S., 2008. Frost formation on superhydrophobic surface under natural convection conditions. International Journal of Heat and Mass Transfer 51, 5975-5982.

[26] Mangel, R. F., Baer, E., 1962. The evaporation of water drops from a "Teflon" surface. Chemical Engineering Science 17 (9), 705-706.

[27] Maynes, D., Crockett, J., 2014. Apparent temperature jump and thermal transport in channles with streamwise rib and cavity featured superhydrophobic walls at constant heat flux. Journal of Heat Transfer 136, 011701. 
[28] Maynes, D., Jeffs, K., Woolford, B., Webb, B. W., 2007. Laminar fully-developed flow in a microchannel with hydrophobic surface patterned micro-ribs oriented parallel to the flow direction. Phys. Fluids 19, 093603.

[29] Maynes, D., Johnson, M., Webb, B. W., 2011. Free-surface liquid jet impingement on rib patterned superhydrophobic surfaces. Phys. Fluids 22, 052104 .

[30] McHale, G., Aqil, S., Shirtcliffe, N., Newton, M., Erbil, H., October 2005. Analysis of Droplet Evaporation on a Superhydrophobic Surface. Langmuir 21 (24), 11053-11060.

[31] Miljkovic, N., Enright, R., Wang, E. N., 2012. Effect of droplet morphology on growth dynamics and heat transfer during condensation on superhydrophobic nanostructured surfaces. ACS Nano 6 (2), 1776-1785.

[32] Ou, J., Perot, B., Rothstein, J., November 2004. Laminar drag reduction in microchannels using ultrahydrophobic surfaces. Physics of Fluids 16 (12), 4635.

[33] Ou, J., Rothstein, J., October 2005. Direct velocity measurements of the flow past drag-reducing ultrahydrophobic surfaces. Physics of Fluids 17 (10), 103606.

[34] Park, J., Moon, J., 2006. Control of colloidal particle deposit patterns within picoliter droplets ejected by ink-jet printing. Langmuir 22 (8), 3506-3513.

[35] Picknett, R. G., Bexon, R., 1977. The evaporation of sessile or pendant drops in still air. Journal of Colloid and Interface Science 61, 336-350.

[36] Prince, J. F., Maynes, D., Crockett, J., 2012. Analysis of laminar jet impingement and hydraulic jump on a horizontal surface with slip. Phys. Fluids 24, 102103.

[37] Prince, J. F., Maynes, D., Crockett, J., 2014. Jet impingement and the hydraulic jump on horizontal surfaces with anisotropic slip. Phys. Fluids 26, 042104 . 
[38] Rioboo, R., Voue, M., Vaillant, A., Coninck, J. D., 2008. Drop impact on porous superhydrophobic polymer surfaces. Langmuir 24, 14074.

[39] Sefiane, K., Bennacer, R., 2011. An expression for droplet evaporation incorporating thermal effects. Journal of Fluid Mechanics 667, 260-271.

[40] Shastry, A., Case, M. J., Bohringer, K. F., 2006. Directing droplets using microstructured surfaces. Langmuir 22, 6161-6167.

[41] Shin, D. H., Lee, S. H., Choi, C. K., Retterer, S., 2010. The evaporation and wetting dynamics of sessile water droplets on submicron-scale patterned silicon hydrophobic surfaces. J. Micromech. Microeng. 20, 055021.

[42] Shin, D. H., Lee, S. H., Jung, J. Y., Yoo, J. Y., 2009. Evaporating characteristics of sessile droplet on hydrophobic and hydrophilic surfaces. Microelectronic Engineering 86 (4), 1350-1353.

[43] Sobac, B., Brutin, D., November 2011. Triple-Line Behavior and Wettability Controlled by Nanocoated Substrates: Influence on Sessile Drop Evaporation. Langmuir 27 (24), 14999-15007.

[44] Sobac, B., Brutin, D., 2012. Thermal effects of the substrate on water droplet evaporation. Physical Review E 86, 021602.

[45] Tsai, P., Pacheco, S., Pirat, C., Lefferts, L., Lohse, D., 2009. Drop impact upon micro- and nano-structured superhydrophobic surfaces. Langmuir 25 (50), 12293.

[46] Varanasi, K. K., Deng, T., Smith, J. D., Hsu, M., Bhate, N., 2010. Frost formation and ice adhesion on superhydrophobic surfaces. Applied Physics Letters 97, 234102.

[47] Wang, Z., Lopex, C., Hirsa, A., Koratkar, N., 2007. Impact dynamics and rebound of water droplets on superhydrophobic carbon nanotube arrays. Applied Phys Letters 91, 023105.

[48] Watanabe, K., Udagawa, Y., Udagawa, H., 1999. Drag reduction of newtonian fluid in a circular pipe with a highly water-repellent wall. Journal of Fluid Mechanics 381, 225-238. 
[49] Woolford, B., Maynes, D., Webb, B. W., 2009. Liquid flow through microchannels with grooved walls under wetting and superhydrophobic conditions. Microfluid Nanofluid 7, 121-135.

[50] Xia, D., Brueck, S. R. J., 2008. Strongly anisotropic wetting on onedimensional nanopatterned surfaces. Nano Lett. 8 (9), 2819-2824.

[51] Zhang, X., Tan, S., Zhao, N., Guo, X., Zhang, X., Zhang, Y., Xu, J., October 2006. Evaporation of Sessile Water Droplets on Superhydrophobic Natural Lotus and Biomimetic Polymer Surfaces. ChemPhysChem 7 (10), 2067-2070. 